Stoa

Vol. 3, No. 5, 2012, pp. 109-136

ISSN 2007-1868

\title{
RECONSTRUCGIÓN ESTRUCTURALISTA DE LAS TEORÍAS TERMODINÁMICAS DE RUDOLF J. CLAUSIUS
}

\author{
C. Ulises Moulines \\ Ludwig-Maximilians-Universität \\ München
}

Resumen: Una forma particular de cambio científico es lo que puede denominarse "la cristalización de una teoría". Se trata de una forma que no es ni 'ciencia normal' ni 'ciencia revolucionaria' en el sentido de Kuhn. Se trata de un tipo de cambio paulatino aunque fundamental en el marco teórico de una disciplina a lo largo de un periodo relativamente largo. Un ejemplo de ello es la emergencia gradual de la termodinámica fenomenológica a mediados del siglo xix. En el curso de ese proceso, los artículos de Clausius de 1850 y 1854 tuvieron un papel crucial. En el presente ensayo se analiza su estructura formal por medio de la metodología de reconstrucción estructuralista. Se identificarán aquí tres "redes teóricas" diferentes en los escritos de Clausius, que corresponden a otros tantos pasos en la cristalización de la termodinámica. La reconstrucción que aquí se ofrece también explicita el modo como las ideas de Clausius evolucionaron durante el periodo histórico en consideración.

PAlabRas Clave: termodinámica · cristalización · estructuralismo · redes teóricas $\cdot$ relaciones interteóricas

Abstract: A particular form of scientific change is what may be called "the crystallization of a theory". This is neither 'normal' nor 'revolutionary science' in Kuhn's sense. It represents a kind of piecemeal but nevertheless fundamental change of the framework of a discipline during a relatively long period of time. An example of it is the gradual emergence of phenomenological thermodynamics in the middle of the 19th century. In this process, Clausius's papers of 1850 and 1854 played a crucial role. Their formal structure is analyzed in the present essay by employing the structuralist reconstruction methodology. Three different "theory-nets" will be identified in the work of Clausius, corresponding to as many steps in the crystallisation of thermodynamics. This reconstruction will also make explicit the way Clausius's ideas evolved during the historical period under consideration. 
KeYwORDS: thermodynamics - crystallization · structuralism · theory-nets · intertheoretical relations

\section{Introducción}

El programa estructuralista de reconstrucción de teorías científicas permite no sólo elucidar la estructura interna de una teoría previamente bien identificada en toda su complejidad, sino también identificar distintas teorías que aparentemente constituyen una sola, así como explicitar su evolución a través del tiempo histórico. En el presente ensayo se aplica la metodología estructuralista al análisis de dos escritos de Rudolf Clausius sobre fenómenos termodinámicos que en la historiografía convencional se interpretan como la presentación de la primera gran teoría termodinámica fenomenológica cuando en realidad, como veremos, se trata de tres teorías distintas cada una con su propia identidad. La reconstrucción nos permitirá asimismo retrazar la forma en que las ideas de Clausius evolucionaron durante un breve periodo histórico.

En la década de 1850, después de que la teoría del calórico se había derrumbado definitivamente y cuando aún no se había establecido un paradigma alternativo para dar cuenta de los fenómenos térmicos en general, Rudolf Clausius publicó tres artículos pioneros en este campo: Über die bewegende Kraft der Wärme und die Gesetze, welche sich daraus für die Wärmelehre selbst ableiten lassen en 1850, Über eine veränderte Form des zweiten Hauptsatzes der mechanischen Wärmetheorie en 1854, y Über die Art der Bewegung, die wir Wärme nennen en $1857 .{ }^{1}$ La diferencia de enfoque entre el último ensayo y los dos primeros es evidente para cualquier lector mínimamente informado y así ha sido vista por todos los historiadores que se han ocupado del tema: en el artículo de 1857 Clausius sienta las bases para la teoría cinética de los gases, que más adelante se incluirá en la mecánica estadística, mientras que los trabajos de 1850 y 1854 no presuponen (casi) ninguna hipótesis sobre la constitución microscópica de los sistemas físicos considerados; por ello se les suele considerar como la primera exposición de la termodinámica fenomenológica. Veremos, sin embargo, gracias al análisis estructuralista, que

\footnotetext{
${ }^{1}$ En su traducción al español: Sobre la fuerza motriz del calor y las leyes que de ella pueden ser derivadas para la teoría del calor; Sobre una forma modificada del segundo principio de la teoría mecánica del calor, y Sobre el tipo de movimiento que llamamos calor, respectivamente.
} 
estos dos artículos contienen en conjunto tres teorías fenomenológicas distintas, si bien, claro está, relacionadas. A la primera teoría, la construida en 1850, la llamaremos, para abreviar, "Clausius 1"; a las otras dos, expuestas en 1854, las llamaremos respectivamente "Clausius 2.1" y "Clausius 2.2".

\section{Reconstrucción de "Clausius 1"}

La dificultad principal a la hora de reconstruir coherentemente la teoría "Clausius 1" proviene de que el artículo en que Clausius la expone no tiene el carácter de un tratado sistemático, sino más bien el de un reporte de investigación, en el cual él -todavía poco conocido como científico- intenta convencer al lector paso a paso de la pertinencia de sus ideas.

El escrito se presenta dividido en dos partes, tituladas respectivamente "Consecuencias del principio de la equivalencia de calor y trabajo", y "Consecuencias del principio de Carnot en conexión con el anterior". ${ }^{2}$ Dada la forma de exposición de Clausius, podría pensarse que se introducen aquí dos principios diferentes y lógicamente independientes: (1) El principio de la equivalencia calor-trabajo, y (2) (lo que Clausius considera que es) El principio de Carnot; a saber, en la propia interpretación de Clausius, que "cada vez que el trabajo produce calor, y no se produce una transformación permanente en el estado del cuerpo que actúa, cierta cantidad de calor pasa de un cuerpo caliente a uno frío" (p. 4). Ahora bien, creo que esta interpretación, que se ha prolongado hasta el día de hoy, acerca de lo que hace Clausius, es errónea, tanto desde el punto de vista de la reconstrucción lógica como desde un punto de vista histórico inmanente a sus ideas. De hecho, el propio Clausius subraya en varias ocasiones que los dos principios están "íntimamente vinculados". Veremos en la reconstrucción (inmanente) de su trabajo que lo más adecuado es interpretar ambos "principios" como dos especificaciones de un solo principio más fundamental y general, implícito en la exposición de Clausius.

Es sabido que al emprender la reconstrucción estructuralista de una teoría cualquiera la primera pregunta que debemos plantearnos es:

${ }^{2}$ Aquí y en lo que sigue doy directamente la traducción al castellano, lo más fiel posible, del texto original en alemán. 
¿cuál es la forma de los modelos (potenciales) de la teoría? Una respuesta adecuada a esta pregunta presupone haber determinado previamente la ontología básica de la teoría en cuestión (los objetos de los que trata) y las relaciones (y/o magnitudes) definidas sobre esa ontología que son absolutamente necesarias para expresar las leyes de la teoría. Veámoslo en el caso que nos ocupa.

\subsection{La ontología básica}

Clausius habla una y otra vez de cuerpos (en tanto entidades concretas localizadas espacio-temporalmente y que están en contacto mutuo o bien tienen "partes componentes"). En algunos casos particulares siente además la necesidad de distinguir las sustancias (químicas) a las que pertenecen esos cuerpos; pero ello sólo lo hace cuando entra en algunas especializaciones de la teoría. Ello significa que el "compromiso ontológico" con diferentes tipos de cuerpos ocurre sólo en el estadio de las especializaciones (sobre todo para los gases ideales). Finalmente, Clausius habla con frecuencia del hecho de que esos cuerpos se encuentran en un cierto estado, o que "modifican su estado", o también que se da una "transición" de un estado a otro dentro del mismo cuerpo, sin presuponer en todo ello una especificación de dichos "estados". Por consiguiente, la ontología básica de cada modelo de la teoría estará constituida por:

Un conjunto finito de cuerpos: $K=\left\{k_{1}, k_{2}, \ldots\right\}$;

Una secuencia continua de estados: $Z \cong \mathbb{R}^{+}$.

\subsection{Las magnitudes primitivas}

Clausius necesita cierto número de magnitudes primitivas (no definibles) para formular sus leyes. Ahora bien, en este punto la metodología estructuralista "ortodoxa" se enfrenta a un problema: según dicha metodología, el conjunto de magnitudes que hay que postular debe ser el mismo en todos los modelos de la teoría. El problema con "Clausius 1" es que esta teoría trata de dos tipos, bastante heterogéneos, de aplicaciones intencionales: los gases ideales por un lado y los vapores al máximo de su densidad por otro. Las leyes especiales para uno y otro tipo de aplicación intencional contienen, sin duda, algunas magnitudes en común, pero otras magnitudes son específicas del tipo de aplicación. Ello implica formalmente que, en la construcción de la 
red teórica que ha de representar "Clausius 1", hay que partir de ciertas magnitudes básicas para los modelos del elemento teórico básico y posteriormente añadirles ciertas magnitudes específicas.

Las magnitudes básicas comunes a la red son:

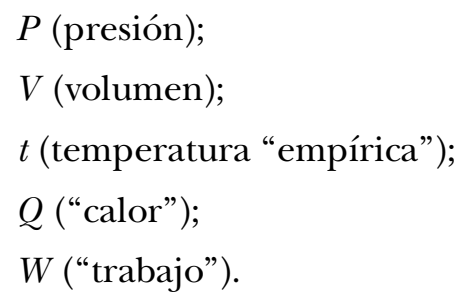

No cabe duda de que $Q$ y $W$ son conceptos fundamentales en "Clausius 1" -digan lo que digan los libros de texto y las exposiciones históricas convencionales, que pretenden que "calor" ya no era un concepto fundamental para Clausius. Él habla todo el tiempo de estas magnitudes, ellas aparecen explícitamente en las leyes fundamentales (y las especiales) y son funciones diferenciables (aunque Clausius, al igual que la mayoría de físicos hasta el día de hoy, no especifique respecto de qué parámetro son diferenciables -sin duda lo son respecto a los estados por los que atraviesa un cuerpo).

Las magnitudes que hay que añadir para formular las especializaciones son, respectivamente:

Para los gases ideales (abreviados en lo que sigue simplemente como "gases"):

- $R$ (un parámetro específico para cada sustancia gaseosa, que depende del peso específico de la sustancia (p. 11); no se trata pues de nuestra constante universal " $R$ ”, aunque está emparentada con ella);

- $C_{v}$ (el calor específico a volumen constante);

- $C_{p}$ (el calor específico a presión constante);

- U (una función "anónima" o "arbitraria", ("willkürlich" es la palabra alemana utilizada por Clausius); se trata sin duda de lo que luego se denominará la "energía interna").

Para los vapores al máximo de su densidad (que abreviaremos por "vapores"): 
- $s$ (el volumen del vapor a su máxima densidad);

- $\sigma$ (el volumen del líquido a la densidad máxima del vapor);

- $m$ (la masa de líquido que se convierte en vapor);

- $c$ (el calor específico del líquido);

- $h$ (otra función "anónima”, requerida para formular ecuaciones diferenciales, de la que Clausius sólo dice que debe depender de la temperatura del vapor $-c f r$. p. 21).

Además de los dominios básicos de entidades y de las magnitudes primitivas, "Clausius 1" requiere de un conjunto auxiliar, $\mathbb{R}$, para expresar los valores, no necesariamente racionales, de las magnitudes, y de dos constantes universales, $A$ (la constante de proporcionalidad calor-trabajo) para todas las aplicaciones intencionales y $a$ (una constante "anónima" para los gases, de la que hoy día diríamos que está relacionada con la temperatura absoluta y que es de interés sólo para la especialización de los gases ideales). Para estas dos constantes Clausius propone respectivamente los valores numéricos (más o menos hipotéticos en su momento y de acuerdo con los resultados experimentales de Joule y otros investigadores que él cita): ${ }^{3}$

$$
A=\frac{1}{421} ; \quad a=273 .
$$

Discutamos brevemente en este punto la cuestión de la $T$-teoricidad; aunque no puedo dar aquí un argumento definitivo, parece evidente que $P, V, t, R, s$ y $\sigma$ son $T$-no-teóricas, puesto que sus valores eran ya conocidos a través de teorías y métodos previos a "Clausius 1". Por el otro lado, $U$ y $h$ son claramente $T$-teóricas: el propio Clausius deja claro que estas funciones "anónimas" sólo tienen sentido dentro del marco de la teoría que está proponiendo, y sus valores sólo se pueden determinar asumiendo las leyes en las que aparecen. A modo de hipótesis, y sin que yo pueda dar un argumento definitivo al respecto, sugiero que en esta teoría $Q, W, C_{v}, C_{p}$ y $c$ también deben ser consideradas $T$-teóricas.

\footnotetext{
${ }^{3}$ Para la reconstrucción formal de la teoría no es relevante indicar los valores numéricos concretos de estas constantes.
} 
Podemos definir ahora los modelos potenciales y potenciales parciales de "Clausius 1". A la teoría ya reconstruida la denominaremos a partir de ahora oficialmente " $T[\mathbf{C L} \mathbf{1}]$ ":

Definición $1 M_{p}[\mathbf{C L} \mathbf{1}]: x \in M_{p}[\mathbf{C L} \mathbf{1}]$ si y sólo si $\exists K, Z, P, V, t, Q, W$, $A$ tales que:

(0) $x=\langle K, Z, \mathbb{R}, A, P, V, t, Q, W\rangle$;

(1) $K$ es un conjunto finito, $K \neq \varnothing$;

(2) $Z \cong \mathbb{R}$;

(3) $A \in \mathbb{R}$;

(4) $P: K \times Z \mapsto \mathbb{R}$ y $P$ es diferenciable con respecto al segundo argumento;

(5) $V: K \times Z \mapsto \mathbb{R}$ y $V$ es diferenciable con respecto al segundo argumento;

(6) $t: K \times Z \mapsto \mathbb{R}$ y $t$ es diferenciable con respecto al segundo argumento;

(7) $Q: K \times Z \mapsto \mathbb{R}$ y $Q$ es diferenciable con respecto al segundo argumento;

(8) $W: K \times Z \mapsto \mathbb{R}$ y $W$ es diferenciable con respecto al segundo argumento.

Definición $2 M_{p p}[\mathbf{C L} 1]: x \in M_{p p}[\mathbf{C L} 1]$ si y sólo si $\exists K, Z, P, V, t, Q, W, A$ tales que:

(0) $x=\langle K, Z, \mathbb{R}, A, P, V, t\rangle$;

(1) $\langle K, Z, \mathbb{R}, A, P, V, t, Q, W\rangle \in M_{p}[\mathbf{C L} \mathbf{1}]$.

Después de haber determinado el marco conceptual de una teoría $\left(M_{p}\right.$ y $\left.M_{p p}\right)$, el siguiente paso en una reconstrucción estructuralista es, como sabemos, la determinación de los modelos actuales $(M)$ del elemento teórico básico. Ello significa que hay que identificar la ley o las leyes fundamentales de la teoría. Una hipótesis metateórica del estructuralismo es que en las teorías con una cierta complejidad interna habrá una sola ley fundamental o "principio-guía" con escaso o nulo 
contenido empírico, que funciona más bien como "esquema". A primera vista la teoría $T[\mathbf{C L} 1]$ parece contravenir esta hipótesis, pues Clausius postula de entrada explícitamente dos axiomas, el de la equivalencia calor-trabajo y el Principio de Carnot; no obstante, voy a argüir en lo que sigue que las apariencias engañan y que la hipótesis metateórica del estructuralismo sigue siendo válida en este caso.

El primer principio, el de la equivalencia calor-trabajo, lo enuncia Clausius de manera semiformal (p. 17) y lo desarrolla en la primera parte del escrito:

$$
\frac{\text { el calor consumido }}{\text { el trabajo producido }}=A
$$

En cuanto al Principio de Carnot, que es relevante para la segunda parte del ensayo, Clausius lo enuncia de manera puramente informal: "a la producción de trabajo en tanto que equivalente le corresponde una mera transmisión de calor de un cuerpo caliente a uno frío" (p. $30)$.

Parecería pues que hay dos principios-guía en $T[\mathbf{C L 1}]$, y no sólo uno; no obstante, es de notar que, en la formulación del Principio de Carnot que acabamos de citar, Clausius presupone que existe una equivalencia entre trabajo y calor -lo cual es la esencia del primer principio, y de hecho, en otros pasajes del ensayo nuestro autor subraya repetidas veces que ambos principios están "íntimamente vinculados”. Nos enfrentamos pues a dos posibilidades interpretativas: (1) el principio de la equivalencia calor-trabajo es el único principio fundamental y el Principio de Carnot es una especialización del mismo en el sentido estructuralista; (2) los dos principios constituyen de hecho dos aspectos de un solo principio-guía implícito, que puede formularse sintéticamente. Ambas interpretaciones son coherentes con el resto del texto; sin embargo, yo favorezco la segunda porque es muy fácil de formular y porque me parece más acorde con las verdaderas intenciones de Clausius. En efecto, si admitimos que $Q$ y $W$ son funciones diferenciables - lo cual Clausius presupone todo el tiempo- entonces es inmediato reformular ambos principios formalmente: 
Principio de equivalencia:

$$
\frac{d Q(k, z)}{d W(k, z)}=A \in \mathbb{R}
$$

Principio de Carnot:

$$
d W(k, z)>0 \rightarrow d Q(k, z)<0 .
$$

Entonces es inmediato formular una versión sintética de las dos fórmulas precedentes:

$$
d Q(k, z)=-A \cdot d W(k, z), \quad \text { donde } \quad A>0 .
$$

He aquí la ley fundamental de $T[\mathbf{C L 1}]$. El lector observará que, como es típico de las leyes fundamentales, esta fórmula por sí sola es casi vacía de contenido puesto que las magnitudes $Q$ y $W$ por sí solas "no nos dicen nada" (recordemos que son $T$-teóricas). Hay que poner a cada una de ellas en relación con las demás magnitudes fundamentales de la teoría: $P, V, t, Y$; de hecho, en su argumentación Clausius presupone que $Q$ y $W$ son dependientes de dichas magnitudes. En términos formales ello significa que $Q$ y $W$ son funcionales (no especificados en este nivel de la red teórica) de las funciones $P, V, t$.

Las consideraciones precedentes nos permiten definir los modelos actuales de la teoría sin más:

Definición $3 M_{o}[\mathbf{C L 1}]: x \in M_{o}[\mathbf{C L} 1]$ si y sólo si $\exists K, Z, P, V, t, \mathrm{Q}, \mathrm{W}$, $A, f^{Q}, f^{W}$, tales que:

(0) $x=\langle K, Z, \mathbb{R}, A, P, V, t, Q, W\rangle$ y $x \in M_{p}[\mathbf{C L} \mathbf{1}]$

(1) $\forall k \in K \forall z \in Z: Q(k, z)=f^{Q}(P(k, z), V(k, z), t(k, z), \ldots)$;

(2) $\forall k \in K \forall z \in Z: W(k, z)=f^{W}(P(k, z), V(k, z), t(k, z), \ldots)$;

(3) $A>0$;

(4) $\forall k \in K \forall z \in Z: d Q(k, z)=-A \cdot d W(k, z)$.

Podríamos sintetizar las condiciones (1)-(4) en una sola fórmula: (LFCL1) $\forall k \in K \forall z \in Z$ :

$$
\begin{aligned}
d f^{Q}(P(k, z), & V(k, z), t(k, z), \ldots)= \\
& -A d f^{W}(P(k, z), V(k, z), t(k, z), \ldots), \text { para } \quad A>0 .
\end{aligned}
$$


He aquí la ley fundamental de la teoría.

No parece que haya condiciones de ligadura interesantes en este nivel de la red teórica, ni siquiera ligaduras de identidad. En efecto, de acuerdo con la metodología estructuralista usual, las condiciones de ligadura de una teoría $T$ se postulan para las magnitudes $T$-teóricas solamente (pues se supone que las magnitudes $T$-no-teóricas ya han sido "constreñidas" en las teorías subyacentes correspondientes). En nuestro caso esto significaría que habría que introducir ligaduras, al menos de identidad, para $Q$ y $W$, sin embargo, una característica esencial de la interpretación de estas magnitudes en la teoría $T[\mathbf{C L 1}]$ es justamente que un mismo cuerpo puede hallarse en un mismo estado al principio o al final de un proceso determinado (un ciclo), y no obstante, el valor de $Q$ y $W$ para ese estado ser diferente al inicio y al final del proceso. Desde un punto de vista modelo-teórico, ello significa que si cambiamos de modelo, incluso si los cuerpos y los estados considerados son los mismos, ellos pueden mostrar valores distintos de $Q$ y $W$. Por consiguiente,

$$
C[\mathbf{C L L} 1]=\wp\left(M_{p}\right) .
$$

Deberíamos indicar ahora los vínculos de la teoría de Clausius con otras teorías (subyacentes). Una discusión detallada de esta cuestión rompería el marco de este ensayo. Lo que parece claro es que las magnitudes CL1-no-teóricas, $P, V$ y $t$, están vinculadas con teorías que preceden (metodológica e históricamente) a $T[\mathbf{C L} 1]$ : para $P$, con la hidrodinámica; para $V$, con la geometría física y para $t$. . ¿ंcon cuál teoría? Aquí se nos plantea un interesante problema histórico-metodológico, pues parece ser que la única teoría bien articulada en tiempos de Clausius que trataba de la temperatura sistemáticamente era la teoría del calórico... la cual Clausius justamente intentaba superar definitivamente. Dejaremos abierta esta cuestión. En todo caso, por lo dicho parece plausible postular para el elemento teórico básico de $T[\mathbf{C L 1}]$ al menos tres vínculos interteóricos:

$$
L[\text { GL1 }]=\left\{\lambda_{P}(\text { CL1 1, HD }), \lambda_{V}(\text { CL1, GEOM }), \lambda_{t}(\text { GL1 } 1, ?)\right\} .
$$

El último paso en la construcción de un elemento teórico es la determinación (informal) del dominio de aplicaciones intencionales. Al respecto, podemos acudir fácilmente al propio Clausius, quien dice: "[en 
este trabajo] nos queremos limitar a la consideración de los gases permanentes [ideales] y de los vapores al máximo de su densidad" (p. 11). Por consiguiente,

$I[\mathbf{C L 1}]=\{$ gases ideales; vapores al máximo de su densidad $\}$.

Teniendo en cuenta los ejemplos que da Clausius de vez en cuando -aunque hay que reconocer que es más bien parco en dar ejemplos de aplicación de su teoría- es plausible determinar el subdominio paradigmático de aplicaciones intencionales como:

$I_{o}[\mathbf{C L 1}]=\{$ hidrógeno; aire atmosférico; vapor de agua $\}$.

Disponemos ahora de todos los constituyentes para la determinación completa del elemento teórico básico:

$$
T_{o}[\mathbf{C L} \mathbf{1}]=\left\langle M_{p}[\mathbf{C L} \mathbf{1}], M_{p p}[\mathbf{C L 1}], M_{o}[\mathbf{C L} \mathbf{1}], \wp\left(M_{p}\right), L[\mathbf{C L} \mathbf{1}], I[\mathbf{C L} \mathbf{1}]\right\rangle
$$

\subsection{Especializaciones}

Pueden identificarse dos ramas principales de especializaciones en la red teórica de $T[\mathbf{C L 1}$ ]: la que corresponde a las leyes relevantes para los gases ideales y la que corresponde a los vapores a densidad máxima. Como en otros casos de reconstrucción de teorías empíricas en las que aparecen funcionales "esquemáticos", también aquí se obtienen ambas líneas de especialización especificando los funcionales $f^{Q}$ y $f^{W}$ de la ley fundamental.

Para los gases ideales, Clausius se basa en un análisis teórico-empírico de los ciclos de Carnot-Clapeyron, con las especificaciones siguientes:

$$
\begin{aligned}
& f^{W}=\frac{R d V d t}{V} \quad \text { (p. 15); } \\
& f^{Q}=\left(\frac{d}{d t}\left(\frac{d Q}{d V}-\frac{d}{d V} \frac{d Q}{d t}\right) d V d t \quad\right. \text { (p. 17). }
\end{aligned}
$$

Como el propio Clausius reconoce, la segunda especificación es, tanto desde el punto de vista del cálculo diferencial como del significado físico, algo "sospechosa", puesto que la noción misma de "diferencial del calor" es problemática. No obstante, al combinarla con 
la determinación menos dudosa de $f^{W}$ y realizando ciertos cálculos, Clausius llega a la conclusión que se puede llegar a una ecuación diferencial más plausible si postulamos que debe existir una función "arbitraria" (en términos de Clausius), a la que nuestro autor no da en este texto ningún nombre particular aparte de simbolizarla por " $U$ ", la cual permite formular la primera especialización de la red de manera matemáticamente impecable. (Esta " $U$ " es naturalmente "nuestra" energía interna, que seguimos simbolizando hoy igual que Clausius.)

$$
d Q=d U+A \cdot \frac{a+t}{v} \cdot d V
$$

He aquí la especialización más general para los gases ideales. Para reconstruirla dentro del marco de nuestro formalismo hay que observar que ahora necesitamos cinco nuevos conceptos primitivos: $U, R$, $C_{v}, C_{p}$ e, implícitamente, la noción de sustancia gaseosa. Para determinar formalmente esta noción, conviene admitir un conjunto $\Gamma$ de sustancias gaseosas en tanto que objetos "abstractos" (que se podrían caracterizar, si se quiere, como clases de equivalencia química de cuerpos); por razones formales, conviene asimismo introducir una función $\gamma$ que asigna a cada cuerpo $k$ particular la sustancia gaseosa a la cual pertenece. Finalmente, hay que postular una nueva constante numérica $a$.

Por lo que respecta a la cuestión de la $T$-teoricidad, está claro por el modo como Clausius introduce $U$ que esta magnitud es $T$-teórica. Aunque menos evidente, parece también plausible suponer que $C_{v} \mathrm{y}$ $C_{p}$ son así mismo $T$-teóricas, en cambio, $R$ parece ser $T$-no-teórica, pues Clausius la retoma de determinaciones "puramente experimentales" anteriores.

Todo ello significa que ni $M_{p}$ ni $M_{p p}$ pueden ser las mismas estructuras que para el elemento básico: los modelos de esta especialización pueden ser considerados como "superestructuras" respecto de los modelos del elemento teórico básico. Llamando "G" al elemento teórico correspondiente a los gases ideales, tendremos por consiguiente la si- 
guiente relación entre las estructuras respectivas de $\mathbf{G}$ y $\mathbf{C L 1}:^{4}$

$(\alpha)$ Para todo $x=\left\langle K, Z, \Gamma, \gamma, \mathbb{R}, A, a, R, P, V, t, Q, W, U, C_{v}, C_{p}\right\rangle$

$\in M_{p}[\mathbf{G}]$, existe $x^{\prime} \in M_{p}[\mathbf{C L} \mathbf{1}]$ tal que $x^{\prime}<x$.

( $\beta)$ Para todo $x=\left\langle K, Z, \Gamma, \gamma, \mathbb{R}, A, a, R, P, V, t, Q, W, U, C_{v}, C_{p}\right\rangle$

$$
\in M_{p p}[\mathbf{G}] \text {, existe } x^{\prime} \in M_{p p}[\mathbf{C L} \mathbf{1}] \text { tal que } x^{\prime}<x \text {. }
$$

He aquí las nuevas condiciones que caracterizan los nuevos concep$\operatorname{tos} \Gamma, a, R, U, C_{v}, C_{p}$ :

(1) $\Gamma$ es un conjunto finito, $\Gamma \neq \varnothing$;

(2) $\gamma: K \mapsto \Gamma$;

(3) $a \in \mathbb{R}$;

(4) $R: \Gamma \mapsto \mathbb{R}$;

(5) $U: K \times Z \mapsto \mathbb{R}$ y $U$ es una función diferenciable;

(6) $C_{\eta}: \Gamma \mapsto \mathbb{R}$;

(7) $C_{p}: \Gamma \mapsto \mathbb{R}$.

Naturalmente, los demás componentes de los modelos potenciales y potenciales parciales de $\mathbf{G}$ satisfacen las mismas caracterizaciones que en $M_{p}[\mathbf{C L} \mathbf{1}]$. Nos ahorramos la definición explícita de $M_{p}[\mathbf{G}]$ y $M_{p p}[\mathbf{G}]$. En cambio, conviene reconstruir explícitamente $M[\mathbf{G}]$.

Definición 4 M[G]: $x \in M[\mathbf{G}]$ si y sólo si $\exists K, Z, \Gamma, \gamma, P, V, t, Q, W, U$, $R, C_{v}, C_{p}, A, a, f^{Q}, f^{W}$ tales que

(0) $x=\left\langle K, Z, \Gamma, \gamma, \mathbb{R}, A, a, P, V, t, Q, W, U, R, C_{v}, C_{p}\right\rangle \in M_{p}[\mathbf{G}]$;

(1) $x=\langle K, Z, \mathbb{R}, A, P, V, t, Q, W\rangle \in M_{o}[\mathbf{C L} \mathbf{1}]$;

(2) $\forall k \in K \forall z \in Z$ :

$$
d Q(k, z)=d U(k, z)+A \cdot R(\gamma(k)) \cdot a+\frac{t(k, z)}{V(k, z)} \cdot d V(k, z) .
$$

Para Clausius, el segundo sumando de la ecuación (2) representa el "trabajo exterior" realizado por el gas. Se obtiene una especialización de esta especialización cuando suponemos que este trabajo tiene la simple forma " $p d V$ ". En tal caso, por un simple cálculo obtendremos

\footnotetext{
${ }^{4}$ El símbolo “<” debe leerse como “... es subestructura de ...”.
} 
la ley de Gay-Lussac. Llamaremos a esta especialización de la especialización G "GL":

Definición $5 M[\mathbf{G L}]: x \in M[\mathbf{G L}]$ si y sólo si $\exists K, Z, \Gamma, \gamma, P, V, t, Q$, $W, U, A, a, f^{Q}, f^{W}$ tales que

(0) $x=\langle K, Z, \Gamma, \gamma, \mathbb{R}, A, a, P, V, t, Q, W, U\rangle \in M[\mathbf{G}]$;

(1) $\forall k \in K \forall z \in Z$ :

$$
A \cdot R(\gamma(k)) \cdot a+\frac{t(k, z)}{V(k, z)} \cdot d V(k, z)=p(k, z) d V(k, z) .
$$

Nota bene: En el contexto de la teoría de Clausius las especializaciones $\mathbf{G}$ y GL son intensionalmente diferentes pero extensionalmente iguales.

Es interesante observar que estas dos especializaciones, al contrario del elemento teórico básico, cumplen una condición de ligadura de identidad: si dos cuerpos que aparecen en dos modelos diferentes pertenecen a la misma sustancia gaseosa, tienen el mismo valor de $R$ :

$$
\begin{aligned}
\mathbf{C}^{=}{ }_{\mathbf{R}}[\mathbf{G}]: \quad \forall X \in \wp\left(\mathbf{M}_{\mathbf{p}}[\mathbf{G}]\right) \forall x, x^{\prime} \in X \forall k \in K_{x} \forall k^{\prime} \in K_{x}^{\prime}: \\
\gamma_{x}(k)=\gamma_{x}^{\prime}\left(k^{\prime}\right) \rightarrow R_{x}\left(\gamma_{x}(k)\right)=R_{x}^{\prime}\left(\gamma_{x}^{\prime}\left(k^{\prime}\right)\right) .
\end{aligned}
$$

Una especialización ulterior en esta misma línea consiste en la hipótesis molecular" (aunque Clausius no la denomina explícitamente así -él sólo habla de una hipótesis "auxiliar", p. 24) sobre la constitución de los gases ideales: tales gases poseen una constitución interna tal que no realizan "trabajo interior" cuando reciben calor, es decir, todo el trabajo realizado es "trabajo exterior" y por consiguiente $U$ sólo es función de la temperatura. Sin necesidad de definir el predicado conjuntista correspondiente, podemos formular esta nueva especialización así:

$$
\text { [Mol] : } \quad[\mathbf{G}] \wedge \exists \phi \forall k \in K \forall z \in Z(U(k, z)=\phi(t(k, z)) .
$$

A renglón seguido, Clausius postula una hipótesis adicional: que "probablemente", en ese caso, $\phi$ es una función muy simple, determinada por $C_{v}$, y que a su vez nos permite determinar también la expresión de $C_{p}$ :

$\left[\mathbf{M o l}^{\prime}\right]: \quad[\mathbf{G}] \wedge U(k, z)=C_{v}(\gamma) \cdot t(k, z) \wedge C_{p}(\gamma(k))-C_{v}(\gamma(k))=A \cdot R(\gamma)$. 
De $\left[\mathbf{M o l}^{\prime}\right]$ se desprende de inmediato como corolario la "ley de Poisson":

Corolario $1 \forall \gamma \in \Gamma: C_{p}(\gamma) / C_{v}(\gamma)$ es una constante.

La otra rama de las especializaciones de CL1 corresponde a los vapores de densidad máxima. En este caso tenemos dos especificaciones completamente diferentes del calor y del trabajo en la ley fundamental. Pero antes de reformularlas cabe observar que también esta especialización resulta de la expansión modelo-teórica del aparato conceptual básico de la teoría, es decir, que los modelos potenciales del elemento teórico básico son subestructuras de los modelos potenciales de las especializaciones para los vapores: además de los dominios básicos $K$ y $Z$, y de las magnitudes básicas $P, V, t, Q$ y $W$, así como de la constante universal $A$, se requiere un conjunto de sustancias (que son diferentes de los gases ideales), en las cuales podemos distinguir una fase líquida de una fase vaporosa; simbolizaremos este conjunto de sustancias por " $\Pi$ "; al igual que en el caso de $\Gamma$, es conveniente introducir una función de asignación $\pi$ de los cuerpos a las sustancias; además, necesitamos las magnitudes adicionales siguientes:

(1) $s$ (volumen de vapor a la densidad máxima): $\Pi \mapsto \mathbb{R}$;

(2) $\sigma$ (volumen de líquido a la densidad máxima): $\Pi \mapsto \mathbb{R}$;

(3) $m$ (masa de líquido que se convierte en vapor) $: K \times Z \mapsto \mathbb{R}$;

(4) $c$ (calor específico de la fase líquida de cada sustancia): $\Pi \mapsto \mathbb{R}$;

(5) $h$ (una función "anónima", aparentemente dependiente de $t$, pero independiente de la sustancia): $K \times Z \mapsto \mathbb{R}$.

Por consiguiente, los modelos potenciales de la especialización $V$ tendrán la estructura:

Definición $6 M_{p}[\mathbf{V}]: x \in M_{p}[\mathbf{V}]$ si y sólo si: $\exists K, Z, \Pi, \pi, P, V, t, Q, W$, $s, \sigma, m, c, h, A$ tales que:

$$
x=\langle K, Z, \Pi, \pi, \mathbb{R}, A, P, V, t, Q, W, s, \sigma, m, c, h\rangle,
$$

donde $\Pi$ es un conjunto finito y las magnitudes adicionales vienen caracterizadas como lo acabamos de hacer en las condiciones (1)-(5). Nótese que no se trata de funciones diferenciables. 
Definición $7 M_{p p}[\mathbf{V}]$ : Ya he expresado la hipótesis, según la cual parece claro que $s, \sigma$ y $m$ son magnitudes $T$-no-teóricas, mientras que $c$ y $h$ parecen ser $T$-teóricas (la última lo es ciertamente). Ello significa que los modelos potenciales parciales del elemento teórico básico también son subestructuras de los modelos potenciales parciales de las especialización de los vapores:

$$
M_{p p}[\mathbf{C L} \mathbf{1}]<M_{p p}[\mathbf{V}] .
$$

Los modelos actuales de la especialización $\mathbf{V}$ vienen determinados por especificaciones de los funcionales $f^{Q}$ y $f^{W}$ muy diferentes de las que corresponden a los gases ideales:

Definición $8 M[\mathbf{V}]: x \in M[\mathbf{V}]$ si y sólo si: $\exists K, Z, \Pi, P, V, t, Q, W, s$, $\sigma, m, c, h, A$ tales que:

(0) $x=\langle K, Z, \Pi, \pi, \mathbb{R}, A, P, V, t, Q, W, s, \sigma, m, c, h\rangle \in M_{p}[\mathbf{V}]$;

(1) $\forall k \in K \forall z \in Z$ :

$$
\begin{aligned}
d Q(k, z)= & d f^{Q}(P(k, z), V(k, z), t(k, z), \ldots) \\
= & \frac{d V(k, z)}{d t(k, z)}+c(\pi(K))-h(k, z) \cdot d m(k, z) \cdot d t(k, z) \wedge \\
& \wedge d W(k, z)=d f^{W}(P(k, z), V(k, z), t(k, z), \ldots) \\
= & (s(K)-\sigma(K)) \cdot d P(k, z) \cdot d m(k, z) .
\end{aligned}
$$

Utilizando estas determinaciones de $Q$ y $W$ añadidas a la ley fundamental de CL1 (la condición $M_{o}[$ CLL1]-(4); es decir, la equivalencia calor-trabajo), un simple cálculo nos permite derivar el siguiente teorema, que puede considerarse la ley propia de este elemento teórico:

Teorema $1 \quad \forall k \in K \forall z \in Z$ :

$$
\frac{d V(k, z)}{d t(k, z)}+c(\pi(K))-h(k, z)=A \cdot\left(s \left(\pi(k)-\sigma(\pi(k)) \cdot \frac{d p(k, z)}{d t(k, z)} .\right.\right.
$$

La presencia del concepto $\Pi$ en este elemento teórico sugiere la presencia implícita de una ligadura de identidad para la noción de calor específico:

$$
\begin{aligned}
C^{=}{ }_{c}[\mathbf{V}]: \quad \forall A \in \wp\left(M_{p}[\mathbf{V}]\right) \forall x, x^{\prime} \in X \forall k \in K_{x}, \forall k^{\prime} \in k_{x}^{\prime}: \\
\Pi_{x}(k)=\Pi_{x}^{\prime}\left(k^{\prime}\right) \rightarrow c_{x}\left(\Pi_{x}(k)\right)=c_{x}^{\prime}\left(\Pi_{x}^{\prime}\left(k^{\prime}\right)\right)
\end{aligned}
$$


A renglón seguido, Clausius introduce una hipótesis adicional sugerida por los resultados experimentales de Regnault y Pambour, a saber, que la función $h$ siempre es negativa. Ello representaría en consecuencia una especialización adicional a la especialización $\mathbf{V}$ :

$$
\left[\mathbf{V}^{\prime}\right]:[\mathbf{V}] \wedge \forall k \in K \forall z \in Z: h(k, z)<0 .
$$

De nuestra determinación precedente de los elementos teóricos que constituyen la teoría CL1, se desprende que la estructura de su red puede representarse mediante la figura 1, donde las flechas representan las especializaciones sucesivas.

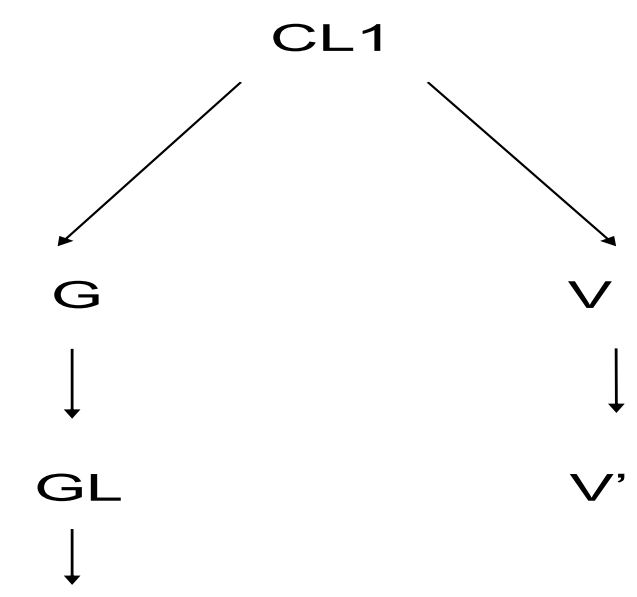

\section{Mol}

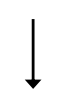

\section{Mol'}

Figura 1: Gráfico de la red teórica $T[\mathbf{C L} \mathbf{1}]$ :

\section{Reconstrucción de "Clausius 2.1"}

El campo de aplicación de las ideas de Clausius en el artículo ya mencionado de 1854, en el que Clausius hace un nuevo intento por sentar las bases de la nueva termodinámica fenomenológica, es a la vez más 
restringido y menos concreto que el de "Clausius 1": se trata explícitamente y (casi) exclusivamente de ciclos ("Kreisprozesse"). Clausius define un ciclo como un proceso de transformación ("Verwandlung"), a través del cual el cuerpo "regresa" al estado inicial (cfr. p. 130).

Otra diferencia notable de este ensayo con respecto a "Clausius 1" reside en el hecho de que nuestro autor trata en él de los "dos principios de la termodinámica" de una manera completamente independiente, es decir, como si se tratara de dos teorías diferentes. Es verdad que, en una observación de pasada (p. 134), hace notar que se podría obtener la formulación más satisfactoria del "Principio de Carnot" (el "segundo principio") poniéndolo en relación con el principio de la equivalencia calor-trabajo (el "primer principio"); pero al mismo tiempo sostiene que ello reduciría la claridad e inteligibilidad del Principio de Carnot. La elección que él hace de la forma de exposición tiene pues un origen más bien "didáctico". En cualquier caso, el hecho es que el artículo de 1854 está dividido en dos partes completamente independientes que habrá que analizar como si fueran dos teorías distintas, que llamaremos "CL2.1" y "CL2.2”. Una y otra parte se presentan con títulos diferentes:

I. "Principio de la equivalencia de calor y trabajo";

II. "Principio de la equivalencia de las transformaciones".

La segunda parte es mucho más larga y argumentada.

Desde un punto de vista formal nos vemos obligados a reconstruir cada una de estas partes como una red teórica diferente, a las que llamaremos " $T$ [CL2.1]" y " $T$ [CL2.2]".

A diferencia de su modo de proceder en el artículo de 1850, ahora Clausius formula el principio de la equivalencia calor-trabajo introduciendo en seguida la función "anónima" $U$ como magnitud fundamental (y, por lo demás, no sólo para el caso de los ciclos); acerca de esta función hace una observación sintomática: se trataría "de una función $U$ que no podemos especificar actualmente, pero de la que por lo menos sabemos que ella está completamente determinada por el estado inicial y el estado final del cuerpo" (p. 130). He aquí un síntoma claro de que $U$ es un concepto $T$-teórico. 
Por consiguiente, Clausius formula la ecuación fundamental de CL2.1 así:

$$
Q=U+A \cdot W
$$

Nota bene: El símbolo " $W$ " (utilizado explícitamente por Clausius) no se refiere ahora al "trabajo total", como en CL1, sino sólo al "trabajo exterior".

La primera especialización considerada por Clausius es aquella en la que el proceso constituye un ciclo. Para ese caso, Clausius escribe: $U=0$. Ahora bien, lo que él presumiblemente quiere decir es que en el caso de los ciclos la suma total de los cambios de $U$ es nula, es decir,

$$
\oint d U=0
$$

Una segunda especialización considerada por Clausius es aquella en la que la presión sobre el cuerpo es la misma en todos los puntos -lo cual es válido, en general, para los gases, los líquidos y algunos sólidos. La fórmula que propone para dar cuenta de este caso es:

$$
d Q=d U+A \cdot p \cdot d V
$$

Hacia el final de esta primera parte del artículo Clausius sugiere que se podrían contemplar otras especializaciones de esta especialización, "aplicando la fórmula a determinadas clases de cuerpos. Para dos de los casos más importantes, a saber, los gases ideales y los vapores al máximo de su densidad, he desarrollado esta aplicación más especial en mi ensayo precedente" (p. 133). Se refiere obviamente a la red $T[\mathbf{C L 1} 1$.

Pasemos ahora a la reconstrucción estructuralista de CL2.1. De entrada, está claro que

$$
M_{p p}[\mathbf{C L 2 . 1}]=M_{p p}[\mathbf{C L} \mathbf{1}]
$$

En cuanto a $M_{p}[$ CL2.1], se trata de una extensión conceptual de $M_{p}[\mathbf{C L 1}]$ puesto que hay que añadir la magnitud $T$-teórica $U$; ahora en tanto que concepto fundamental:

$$
x \in M_{p}[\text { CL2.1 }] \text { si y sólo si } x=\langle K, Z, P, V, t, Q, W, U\rangle,
$$


donde

$$
U: K \times Z \mapsto \mathbb{R}
$$

y $U$ es diferenciable con respecto al segundo argumento.

Formalmente, ello significa que los elementos de $M_{p}[\mathbf{C L} \mathbf{1}]$ son subestructuras de los elementos de $M_{p}$ [CL2.1].

$M_{o}$ [CL2.1] está determinado por las mismas condiciones que $M_{o}[\mathbf{C L 1}]$, salvo por la introducción de un "funcional" adicional para expresar la dependencia de $U$ con respecto a $V$ y $t$ (p. 131) y por la nueva forma de la "ley fundamental":

Definición $9 x \in M_{o}[\mathbf{C 2 . 1}]$ si y sólo si: $\exists K, Z, P, V, t, Q, W, A, U, f^{Q}$, $f^{W}, f^{U}$ tales que:

$$
\begin{aligned}
& \text { (0) } x=\langle K, Z, \mathbb{R}, A, P, V, t, Q, W, U\rangle \text { y } x \in M_{p}[\mathbf{C L 2 . 1}] ; \\
& \text { (1) } \forall k \in K \forall z \in Z: Q(k, z)=f^{Q}(P(k, z), V(k, z), t(k, z)) ; \\
& \text { (2) } \forall k \in K \forall z \in Z: W(k, z)=f^{W}(P(k, z), V(k, z), t(k, z)) ; \\
& \text { (3) } A>0 ; \\
& \text { (4) } \forall k \in K \forall z \in Z: U(k, z)=f^{U}(P(k, z), V(k, z), t(k, z)) ; \\
& \text { (5) } \forall k \in K \forall z \in Z: Q(k, z)=U(k, z)+A \cdot W(k, z) .
\end{aligned}
$$

\subsection{Especializaciones}

La primera especialización concierne los ciclos. De entrada, hay que introducir esta noción mediante una definición auxiliar: en un ciclo, intuitivamente, "el estado final es idéntico al estado inicial". Ahora bien, como formalmente conviene caracterizar un proceso cualquiera como una secuencia de estados isomorfa a un intervalo de números reales, no podemos simplemente postular que el elemento terminal de la secuencia sea estrictamente idéntico al elemento inicial; diremos solamente que es "termodinámicamente idéntico" cuando las funciones pertinentes toman el mismo valor para ambos estados; es decir,

Definición 10 Sea $Z=\left[z_{i}, z_{f}\right]$ un proceso en el sentido de $M_{p}[$ CLL1 $]$. $Z$ es un ciclo si y sólo si:

$$
\begin{aligned}
P\left(k, z_{i}\right) & =P\left(k, z_{f}\right) \quad \text { y } & V\left(k, z_{i}\right) & =V\left(k, z_{f}\right) \quad \text { y } \\
t\left(k, z_{i}\right) & =t\left(k, z_{f}\right) \quad \text { y } & U\left(k, z_{i}\right) & =U\left(k, z_{f}\right) .
\end{aligned}
$$


Definición $11 M[\mathbf{C}]: x \in M[\mathbf{C}]$ si y sólo si : $\exists K, Z, P, V, t, Q, W, A, U$ tales que

(0) $x=\langle K, Z, \mathbb{R}, A, P, V, t, Q, W, U\rangle$ y $x \in M_{o}[\mathbf{C L 2} .1]$;

(1) $Z$ es un ciclo;

(2) $\forall k \in K \forall z \in Z: \oint d U(k, z)=0$.

Otra especialización (independiente de la primera) es aquella en la que $W$ adopta una forma particularmente simple: $P d V$. Esta especialización es aplicable a los gases, a los líquidos y a algunos sólidos. Se la podría caracterizar como el caso de una "presión uniforme" ("PU").

Definición $12 M[\mathbf{P U}]: x \in M[\mathbf{P U}]$ si y sólo si: $\exists K, Z, P, V, t, Q, W, A$, $U$ tales que

(0) $x=\langle K, Z, \mathbb{R}, A, P, V, t, Q, W, U\rangle$ y $x \in M_{o}[\mathbf{C L 2 . 1}]$

(1) $\forall k \in K \forall z \in Z: d Q(k, z)=d U(k, z)+A \cdot P(k, z) \cdot d V(k, z)$.

Según las indicaciones ya citadas del propio Clausius, se podría obtener a partir de aquí la ley de Gay-Lussac para los gases ideales así como las leyes para los vapores; es decir, que podemos incrustar una parte de la red $T[\mathbf{C L 1}]$ en la nueva red $T[$ CL2.1].

\section{Reconstrucción de $T[$ CL2.2]}

Como hemos observado al principio de la sección 3, la teoría CL2.2 está exclusivamente dedicada a la formulación más general posible del "Segundo Principio", con entera independencia del "Primer Principio", y su dominio de aplicaciones intencionales está constituido exclusivamente por ciclos.

De entrada, Clausius da una formulación intuitiva del "Segundo Principio", que considera más general que la que se atribuye generalmente a investigadores precedentes (Carnot, Clapeyron, etc.): "Nunca puede pasar calor de un cuerpo frío a un cuerpo más caliente, a menos que no se presente al mismo tiempo otra modificación que depende de la primera" (p. 134).

La tarea que ahora se plantea Clausius es la de encontrar una formulación matemática apropiada para este principio. En un primer paso, 


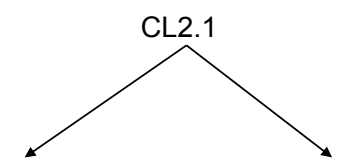

C

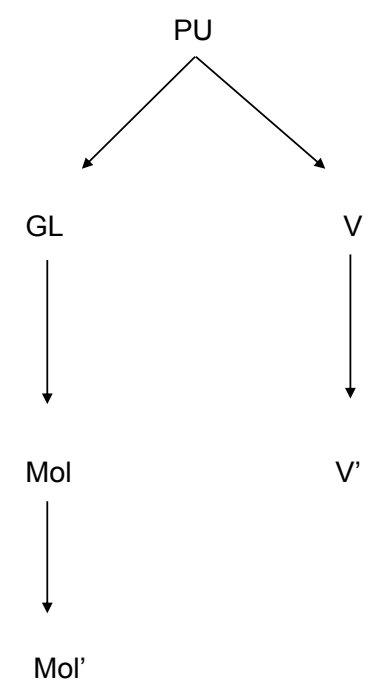

Figura 2: Grafo de la red $T[$ CL2.1]

nuestro autor introduce la noción de "valor de equivalencia" ("Aequivalenzwerth") de una transformación (de trabajo en calor o a la inversa). Su propuesta general es la siguiente:

Valor de equivalencia de $\mathrm{W}: f(t)={ }_{d f} Q \cdot \frac{1}{T(t)}$

Sobre esta nueva función "anónima” $T$, Clausius nos advierte: “ $T$ es la función desconocida de la temperatura, que aparece en los valores de equivalencia" (p. 143). Para nosotros, ello significa obviamente que $T$ ha de ser considerada una magnitud $T$-teórica. Tan sólo al final de su artículo, Clausius emite la hipótesis de que $T$ puede ser interpretada simplemente como siendo la temperatura absoluta.

Antes de pasar a la formulación general del "Segundo Principio", Clausius establece la distinción entre ciclos "reversibles" ("umkehrbar") e "irreversibles". En el texto no da ninguna definición precisa acerca de lo que entiende por "reversible"; aparentemente supone que cual- 
quier lector tendrá una idea clara de lo que ello significa; y a través de una larga argumentación semiformal, llega a la conclusión de que la condición formal de los ciclos reversibles es:

$$
\oint \frac{d Q}{T}=0
$$

Una especialización de esta condición corresponde a la ecuación obtenida por Clapeyron por otra vía:

$$
\frac{d T}{d t} / T=\frac{A}{C},
$$

donde $C$ es la así llamada "función de Carnot".

Para los ciclos irreversibles, Clausius llega a la conclusión de que la condición correspondiente deberá ser

$$
\oint \frac{d Q}{T}>0 .
$$

$\mathrm{Si}$ admitimos la "hipótesis adicional ("Nebenannahme"), que Clausius retoma de Regnault, según la cual "un gas ideal, al expandirse a temperatura constante, absorbe únicamente la cantidad de calor que es necesaria para el trabajo exterior efectuado" (p.153), se obtiene:

$$
\frac{d Q}{d V}=A \cdot P
$$

y si añadimos la ley de Gay-Lussac, obtendremos como teorema:

$$
T=a+t,
$$

donde $a$ "presumiblemente" vale 273. He aquí por qué podemos considerar $T$ como la temperatura absoluta.

Clausius nunca formula explícitamente la ley fundamental de la teoría común a los ciclos reversibles e irreversibles, pero está claro por el contexto que ésta no puede ser otra que

$$
\oint \frac{d Q}{T} \geq 0
$$

Con estos antecedentes de la exposición informal de Clausius pasemos ahora a determinar las diversas estructuras de $T$ [CL2.2] de acuerdo al enfoque estructuralista. 
$M_{p p}[\mathbf{C L 2 . 2 I}]$ es el mismo conjunto que $M_{p p}[\mathbf{C L} \mathbf{1}]$ y por consiguiente que $M_{p p}[\mathbf{C L 2 . 1}]$.

$M_{p}[\mathbf{C L 2 . 2}]$ es una extensión conceptual de $M_{p}[\mathbf{C L}]$ puesto que hay que añadir la nueva magnitud $T$-teórica $T$, la temperatura absoluta. En cambio, en la presente teoría la energía interna $U$ no tiene ningún papel:

$$
x \in M_{p}[\mathbf{C L 2 . 2}] \text { sólo si } x=\langle K, Z, \mathbb{R}, P, V, t, Q, W, T\rangle,
$$

donde

$$
T: K \times Z \mapsto \mathbb{R}
$$

y $T$ es diferenciable con respecto al segundo argumento.

Formalmente esto significa que los elementos de $M_{p}[\mathbf{C L} \mathbf{1}]$ son subestructuras de los elementos de $M_{p}$ [CL2.2].

$M_{o}$ [CL2.2]: Los modelos actuales del elemento básico de esta red teórica vienen determinados por la ley fundamental que expresa la relación entre calor y temperatura absoluta en todo tipo de ciclos. Además, hay que añadir la condición de que para la aplicación de dicha ley hay que presuponer justamente que el dominio básico que representa el proceso es un ciclo en el sentido de la definicion 10.

Definición $13 x \in M_{o}$ [CL2.2] si y sólo si: $\exists K, Z, P, V, t, Q, W, A, T$, $f^{Q}, f^{W}, f^{T}$ tales que:

(0) $x=\langle K, Z, \mathbb{R}, A, P, V, t, Q, W, T\rangle$ y $x \in M_{p}[\mathbf{C L 2}$.2];

(1) $Z$ es un ciclo;

(2) $\forall k \in K \forall z \in Z: Q(k, z)=f^{Q}(P(k, z), V(k, z), t(k, z))$;

(3) $\forall k \in K \forall z \in Z: W(k, z)=f^{W}(P(k, z), V(k, z), t(k, z))$;

(4) $A>0$;

(5) $\forall k \in K \forall z \in Z: T(k, z)=f^{T}(T(k, z))$;

(6)

$$
\forall k \in K \oint \frac{d Q(k, z)}{T(k, z)} \cdot d z \geq 0 .
$$


C. ULISES MOULINES

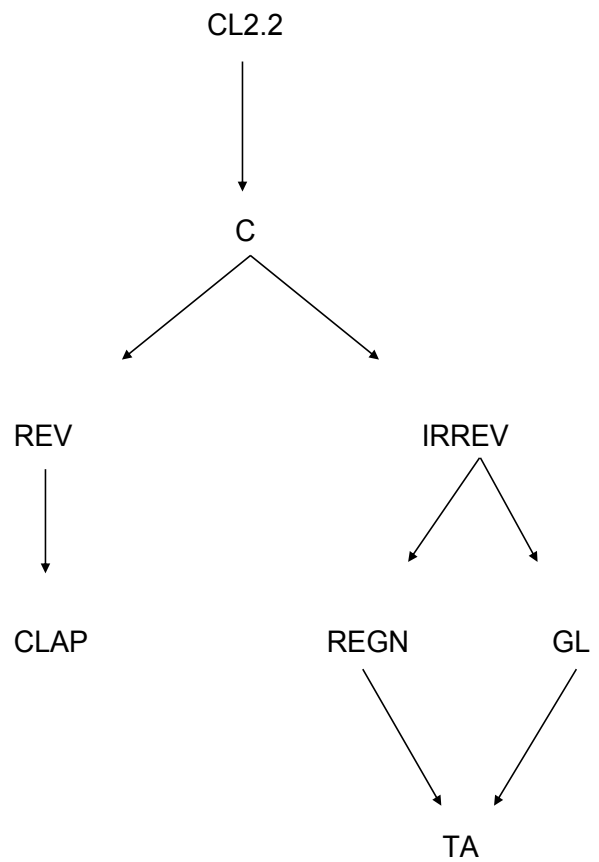

Figura 3: Grafo de la red $T[$ CL2.2]

\subsection{Especializaciones}

Una primera especialización concierne los ciclos reversibles.

Definición $14 M[\mathbf{R E V}]: x \in M[\mathbf{R E V}]$ si y sólo si

(0) $x \in M_{o}$ [CL2.2];

(1) $\forall k \in K$ :

$$
\oint \frac{d Q(k, z)}{T(k, z)} \cdot d z=0 .
$$

Esta especialización se puede especializar a su vez con el fin de obtener la importante ley de Clapeyron, para ello, introducimos una nueva magnitud $T$-teórica, la llamada "función de Carnot", $C$. Nos enfrentamos pues a una extensión conceptual de los modelos precedentes de la red teórica; esto es, los elementos de $M[\mathbf{R E V}]$ devienen subestructuras de los modelos de la especialización de Clapeyron. 
Definición $15 M_{p}[\mathbf{C L A P}]>M_{p}[\mathbf{R E V}]$, donde $x \in M_{p}[\mathbf{C L A P}]$ sólo si $x=\langle K, Z, \mathbb{R}, A, P, V, t, Q, W, T, C\rangle$, con $C: K \times Z \mapsto \mathbb{R}$.

Definición $16 M[$ CLAP $]: x \in M[$ CLAP $]$ si y sólo si

(0) $x=\langle K, Z, \mathbb{R}, A, P, V, t, Q, W, T, C\rangle \in M_{p}[\mathbf{C L A P}]$;

(1) $\langle K, Z, \mathbb{R}, A, P, V, t, Q, W, T\rangle \in M[\mathbf{R E V}]$;

(2) $\forall k \in K \forall z \in Z$ :

$$
\frac{1}{T(k, z)} \cdot \frac{d T(k, z)}{d t(k, z)}=\frac{A}{C(k, z)} .
$$

Otra línea de especialización se refiere a los ciclos irreversibles.

Definición $17 M[$ IRREV] $: x \in M[$ IRREV] si y sólo si

(0) $x \in M[$ CL2.2];

(1) $\forall k \in K$

$$
\oint \frac{d Q(k, z)}{T(k, z)} \cdot d z>0 .
$$

Obtendremos una especialización ulterior al admitir la hipótesis adicional de Regnault antes citada:

Definición $18 M[$ REGN] $: x \in M[$ REGN] si y sólo si

(0) $x \in M[$ IRREV];

(1) $\forall k \in K \forall z \in Z$ :

$$
\frac{d Q(k, z)}{d V(k, z)}=A \cdot P(k, z) .
$$

Otra especialización dentro de esta misma rama consistiría simplemente en incorporar la ley de Gay-Lussac que ya conocemos de $T[\mathbf{C L} 1]: M[\mathbf{G L}]$. Nos ahorramos repetir su formulación aquí.

Si combinamos $M[$ REGN] con $M[\mathbf{G L}]$, es decir, si construimos una especialización común a estas dos especializaciones, a la que podemos denominar $M[\mathbf{T A}]$ (para "temperatura absoluta"), obtendremos el siguiente teorema: 

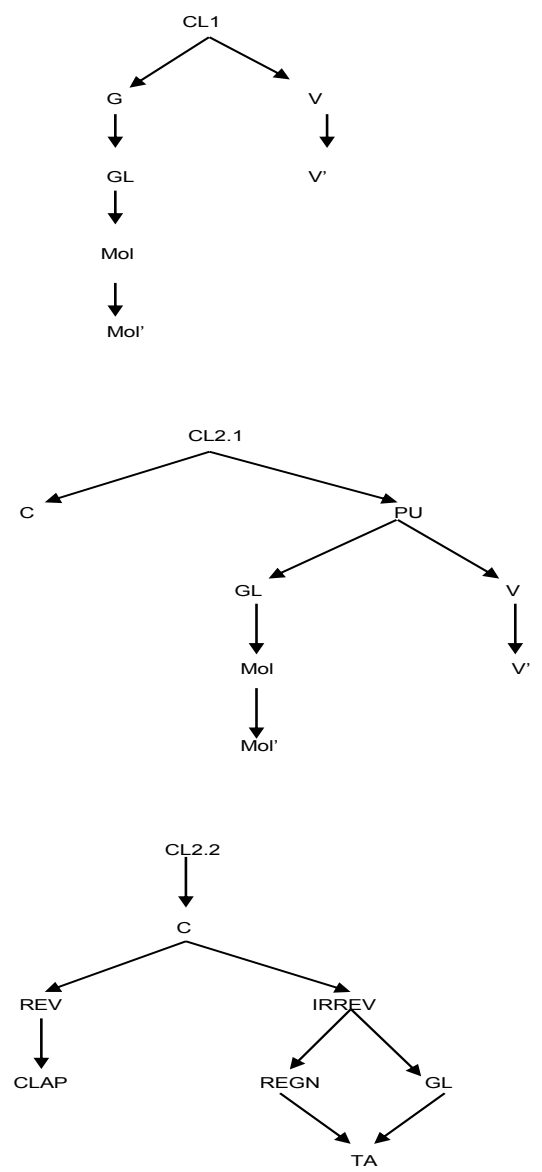

Figura 4: Las relaciones interteóricas entre las redes. 
Teorema 2 Si $x \in M[\mathbf{T A}]$, entonces $\forall k \in K \forall z \in Z$ :

$$
T(k, z)=273+t(k, z) \text {. }
$$

Por consiguiente, la red teórica de $T[$ CL2.2] tiene la forma representada en la figura 3 .

Podemos comparar ahora gráficamente las tres redes teóricas de Clausius reconstruidas hasta aquí: $T[\mathbf{C L} 1], T[C L 2.1]$ y $T[$ CL2.2]. Nótese que algunas de las especializaciones de $T[\mathbf{C L 1}]$ se incrustan en las redes $T[C L 2.1]$ y $T[$ CL2.2]. La figura 4 muestra las relaciones interteóricas entre las redes consideradas.

\section{Referencias}

Clausius, R. J., 1850, Über die bewegende Kraft der Wärme und die Gesetze, welche sich daraus für die Wärmelehre selbst ableiten lassen, Königlich Preussische Akademie der Wissenschaften zu Berlin, Berlín.

- 1854, Über eine veränderte Form des zweiten Hauptsatzes der mechanischen Wärmetheorie, Annalen der Physik und Chemie (Poggendorffs Annalen), tomo 93.

_, 1857 , Über die Art der Bewegung, die wir Wärme nennen, Annalen der Physik und Chemie (Poggendorffs Annalen), tomo 176.

Recibido el 8 de septiembre de 2011

Aceptado el 30 de noviembre de 2011 